\title{
Constraining smoothness parameter and the DD relation of Dyer-Roeder equation with supernovae
}

\author{
Xi Yang, ${ }^{a}$ Hao-Ran Yu ${ }^{a}$ and Tong-Jie Zhang ${ }^{a}$ \\ ${ }^{a}$ Department of Astronomy, Beijing Normal University, \\ Beijing 100875, China \\ E-mail: yangwds@mail.bnu.edu.cn, yu@bnu.edu.cn, tjzhang@bnu.edu.cn
}

\begin{abstract}
Our real universe is locally inhomogeneous. Dyer and Roeder introduced the smoothness parameter $\alpha$ to describe the influence of local inhomogeneity on angular diameter distance, and they obtained the angular diameter distance-redshift approximate relation (Dyer-Roeder equation) for locally inhomogeneous universe. Furthermore, the DistanceDuality (DD) relation, $D_{L}(z)(1+z)^{-2} / D_{A}(z)=1$, should be valid for all cosmological models that are described by Riemannian geometry, where $D_{L}$ and $D_{A}$ are, respectively, the luminosity and angular distance distances. Therefore, it is necessary to test whether if the Dyer-Roeder approximate equation can satisfy the Distance-Duality relation. In this paper, we use Union2.1 SNe Ia data to constrain the smoothness parameter $\alpha$ and test whether the Dyer-Roeder equation satisfies the DD relation. By using $\chi^{2}$ minimization, we get $\alpha=0.92_{-0.32}^{+0.08}$ at $1 \sigma$ and $0.92_{-0.65}^{+0.08}$ at $2 \sigma$, and our results show that the Dyer-Roeder equation is in good consistency with the $\mathrm{DD}$ relation at $1 \sigma$.
\end{abstract}

Keywords: supernova type Ia - standard candles, cosmological perturbation theory, weak gravitational lensing 


\section{Contents}

1 Introduction $\quad 1$

2 Dyer-Roeder equation $\quad 2$

3 Samples 3

4 Analysis and results 4

5 Discussions and Conclusions $\quad 6$

\section{Introduction}

In astronomy, the distance-redshift relation is an important equation. The simplest approximation, cosmological principle, assumes that the universe is homogeneous and isotropic. Using cosmological principle and Einstein equation, the distance-redshift relation for homogeneous universe can be get [1]. However, the real universe at least is locally inhomogeneous, and the inhomogeneity can affect the distance-redshift relation. Therefore, many authors [2-5] discussed this issue, but they did not give a simple formula for this relation. Until 1973, Dyer and Roeder [6] obtained a simple relation (named Dyer-Roeder (D-R) equation) via the method of averaging path.

The $\mathrm{D}-\mathrm{R}$ equation is an angular diameter distance-redshift approximate equation that contains a parameter $\alpha$ (named smoothness parameter) for locally inhomogeneous universe, where $\alpha(0 \leq \alpha \leq 1)$ describes the influence of local inhomogeneity on the angular diameter distance. The detailed information of the D-R equation and $\alpha$ are displayed in Section 2 .

In order to improve the accuracy of the $\mathrm{D}-\mathrm{R}$ equation, the value range of $\alpha$ need to be constrain. Santos et al. [7] used the $182 \mathrm{SNe}$ Ia data of Riess et al. [8] to obtain that $\alpha \geq 0.42(2 \sigma)$. Yu et al. [9] got $\alpha=0.81_{-0.20}^{+0.19}$ at $1 \sigma$ CL via the observational $H(z)$ data, and Busti et al. [10] got $\alpha \geq 0.25(2 \sigma)$ using Union2 SNe Ia data. In this paper we use latest Union2.1 SNe Ia data which contains 580 SNe Ia to constrain $\alpha$, and we get $\alpha=0.92_{-0.32}^{+0.08}$ $(1 \sigma)$ and $0.92_{-0.65}^{+0.08}(2 \sigma)$.

On the other hand, the distance-duality (DD) relation [11, 12] plays a fundamental role in modern cosmology, which reads

$$
\frac{D_{L}}{D_{A}}(1+z)^{-2}=1,
$$

where $D_{L}$ and $D_{A}$ are, respectively, the luminosity and angular diameter distances, and $z$ is the cosmological redshift. Doppler redshift and redshift of tired light theory [1] do not satisfy above expression. This equation is valid for all cosmological models based on Riemannian geometry if photon travels through null geodesic and the photon number is conserved [13], so it should also be valid for an inhomogeneous universe described by Riemannian geometry. The D-R equation is based on Robertson-Walker metric and Einstein equations which are based on Riemannian geometry (see Section 2). So, on the surface, the D-R equation should satisfy the DD relation. However, the D-R equation is obtained via perturbation method, and it just describes the relation of angular diameter distance and redshift, which have nothing 
to do directly with luminosity distance, therefore, it may put a perturbation on the DD relation. So, whether if this equation conflicts with the DD relation need to test. The D-R equation will be modified if the DD relation cannot be satisfied. Fortunately, our results should that the D-R equation is in good consistency with the DD relation. In following paragraph, methods of testing the DD relation are introduced.

A series of works have introduced the parameter $\eta(z)$ to tested the DD relation [14-16], i.e.,

$$
\frac{D_{L}}{D_{A}}(1+z)^{-2}=\eta(z)
$$

and there are generally two ways to test it's validity. The first way to constrain $\eta$ is to combine the observed results of $D_{L}$ and $D_{A}$ both from observations [14,17-19]. This method is popular because it is cosmological model-independent. Holanda et al. [14] artificially assumed that $\eta(z)$ takes two forms, i.e., $\eta(z)=1+\eta_{0} z$ or $\eta(z)=1+\eta_{0} z /(1+z)$, and got $\eta_{0}=-0.28_{-0.44}^{+0.44}$ $(2 \sigma \mathrm{CL})$ for the $D_{A}$ samples of elliptical model [20]. Therefore, their results just satisfy the DD relation at $2 \sigma$ CL for elliptical model. In subsequent papers, Li et al. [18] and Meng et al. [19] obtained the conclusion that DD relation can be accommodated at the $1 \sigma$ CL for the elliptical model. In short, these cosmological model-independent tests suggest that the deviation of $\eta$ from 1 is minor.

Therefore, these conclusions can at least denote that the observed redshift is mainly generated by the expansion of universe, because other kinds of redshift do not satisfy Equation (1.1). So these results can strictly rule out some exotic cosmological models. For example, tired light theory [1] assumes that our universe is basically static and the photons just suffer a loss of energy while they are traveling to us, so the redshift naturally increasing with the distance. This theory concludes that $D_{L}(1+z)^{-1 / 2} / D_{A}=1$. Lima et al. [21] proposed that the small deviation of $\eta$ may indicate that some breaks on fundamental physical theories.

The second way to test DD relation is to combine the observational results of $D_{L}$ (or $D_{A}$ ) with theoretical results of $D_{A}$ (or $D_{L}$ ) for a given cosmological model $[15,16,22,23]$. Obviously, analyses obtained from this way are just valid for the given cosmological model, so this method have too much limitation.

In this paper, we adopt the second method to constrain $\eta$ and $\alpha$, i.e., we combine the observed values of $D_{L}(z)$ from supernovae data with the theoretical values of $D_{A}(z)$ from Dyer-Roeder (D-R) equation to constrain $\alpha$ and $\eta$. Because in this way, our aim can be achieved. Our aim is to constrain $\alpha$ and test whether if the D-R equation satisfies the DD relation.

This paper is organized as follows: In Section 2, we present the D-R equation. Section 3 briefly introduces the sample of SNe Ia data: Union2.1. The analysis methods and results are presented in Section 4. Finally we give the discussions and conclusions in Section 5.

\section{Dyer-Roeder equation}

We consider a light ray which comes to us from a far object propagating along a tube of density $\left(\rho_{\text {int }}=\alpha \rho_{0}\right)$ in a background homogeneous universe of density $\rho_{0}$. According to optical scalar equations [24], the angular diameter distance $D_{A}$ satisfies the following formula:

$$
\frac{\mathrm{d}^{2} D_{A}}{\mathrm{~d} s^{2}}=-\left(|\sigma|^{2}+\frac{1}{2} R_{\alpha \beta} k^{\alpha} k^{\beta}\right)
$$

where $s, \sigma, k^{\alpha}$, and $R_{\alpha \beta}$ are, respectively, the affine parameter, the shear of the light bundle, the vector tangent to the light ray, and the Ricci tensor. For symmetry, the shear $\sigma=$ 
0. Combining the Robertson-Walker metric and Einstein equations, one can obtain the distance-relation equation (D-R equation) for a locally inhomogeneous and flat-space $\Lambda$ CDM cosmological model:

$$
\frac{\mathrm{d}^{2} D_{A}}{\mathrm{~d} z^{2}}+\mathcal{P} \frac{\mathrm{d} D_{A}}{\mathrm{~d} z}+\mathcal{Q} D_{A}=0
$$

where,

$$
\begin{gathered}
\mathcal{P}=\frac{\frac{7}{2} \Omega_{M}(1+z)^{3}+2\left(1-\Omega_{M}\right)}{\Omega_{M}(1+z)^{4}+\left(1-\Omega_{M}\right)(1+z)}, \\
\mathcal{Q}=\frac{\frac{3}{2} \alpha \Omega_{M}}{\Omega_{M}(1+z)^{2}+\left(1-\Omega_{M}\right)(1+z)^{-1}} .
\end{gathered}
$$

Here the initial conditions are

$$
\left\{\begin{array}{l}
D_{A}(0)=0, \\
\left.\frac{\mathrm{d} D_{A}}{\mathrm{~d} z}\right|_{z=0}=\frac{c}{H_{0}},
\end{array}\right.
$$

where $c$ and $H_{0}$ are the speed of light and Hubble constant respectively. The smooth parameter $\alpha$ is defined as

$$
\alpha=\frac{\rho_{\text {int }}}{\rho_{0}},
$$

where $\rho_{\text {int }}$ is the mean density of intergalactic matter in the universe, while $\rho_{0}$ is the mean density of the whole universe, so $\alpha \in[0,1]$. When $\alpha=0$, it means all the matter clustered into stars, galaxies, while $\alpha=1$ means the universe is totally homogeneous. Therefore, the smooth parameter should satisfy $0<\alpha<1$ in the real universe, and $D_{A}$ is a decreasing function of $\alpha$ [25]. Obviously, the smooth parameter should be different at various epoch of the universe [7]. The functional forms of $\alpha(z)$ have been discussed in Ref. [25-29]. However, we will regard $\alpha$ as a constant because there are no convinced functional forms for $\alpha(z)$. This equation have no analytical solution [30-32], but one can get its approximate solution [33].

\section{Samples}

We choose 580 SNe Ia data of Union2.1 $1^{1}$ [34] which cover the redshift interval $0.015 \leq z \leq$ 1.414, to constrain the parameters $\alpha$ and $\eta$. The Union2.1 compilation is an updated version of Union2 [35] compilation by adding new SNe data from the Hubble Space Telescope Cluster Survey to Union2 compilation. All $580 \mathrm{SNe}$ of Union2.1 were fit using the light curve fitter SALT2 [36].

The SALT2 fitter fits all SNe Ia with three parameters. The three parameters are $m_{B}^{\max }$, $x_{1}$, and $c_{1} . m_{B}^{\max }$ is the integrated B-band flux at maximum light. $x_{1}$ is the deviation from the average light-curve shape, and $c_{1}$ is the deviation from the mean $\mathrm{SNe} \mathrm{Ia} \mathrm{B}-\mathrm{V}$ color. The linear combination of the above parameters forms the empirical formula of distance modulus:

$$
\mu_{B}=m_{B}^{\max }+\alpha x_{1}-\beta c_{1}-M_{B},
$$

where $\alpha$ and $\beta$ are constant coefficients, and $M_{B}$ is absolute B-band magnitude of an SNe Ia. $m_{B}^{\max }, x_{1}$, and $c_{1}$ are got by fitting the light curves of SNe Ia. Therefore, the distance modulus fitted by SALT2 have three unknown parameters $\alpha, \beta$, and $M_{B}$, i.e., $\mu=\mu\left(\alpha, \beta, M_{B}\right)$.

\footnotetext{
${ }^{1}$ http://supernova.1bl.gov/Union
} 
The best fitted values of $\alpha, \beta$, and $M_{B}$ are got by $\chi^{2}$-minimization method with a given cosmological model, and then, the best fitted values of distance modulus $\mu\left(\alpha, \beta, M_{B}\right)$ is got. It is important to note that the distance modulus $\mu$ of SNe Ia from Union2.1 is dependent on Hubble constant $H_{0}$, because the given cosmological model has $H_{0}$, which will bring $H_{0}$ into $M_{B}$ if one use $\chi^{2}$-minimization. In Union2.1, they chose $H_{0}=70 \mathrm{kms}^{-1} \mathrm{Mpc}^{-1}$ to get $M_{B}$ and $\mu$ (see Equation (4) and Table 6 in Ref. [34]). So, in this paper, we must choose $H_{0}=70 \mathrm{kms}^{-1} \mathrm{Mpc}^{-1}$ in D-R equation to constrain $\alpha$ and $\eta$.

\section{Analysis and results}

Firstly, we get the numerical solution of $D_{A}$ from Equation (2.2a):

$$
D_{A}=D_{A}\left(H_{o}, \Omega_{M}, \alpha ; z\right) .
$$

Combining Equation (1.2) with above expression, we obtain

$$
D_{L}=\eta(1+z)^{2} D_{A}\left(H_{o}, \Omega_{M}, \alpha ; z\right)=D_{L}\left(H_{o}, \Omega_{M}, \alpha, \eta ; z\right) .
$$

The distance modulus-luminosity distance relation is

$$
\mu=-5+5 \lg D_{L},
$$

where $u$ is distance modulus. Substituting Equation (4.1b) into Equation (4.1c), we get

$$
\mu=\mu\left(H_{o}, \Omega_{M}, \alpha, \eta ; z\right) .
$$

In order to constrain $\alpha$ and $\eta$, we use $\chi^{2}$ minimization and plot the contours on the $(\alpha, \eta)$ plane. The contours of the confidence level of $68.3 \%$ and $95.4 \%$ are determined by two parameter levels 2.30 and 6.18 respectively. For each parameter, the confidence of $68.3 \%(1 \sigma)$ and $95.4 \%(2 \sigma)$, are determined by one parameter levels 1.00 and 4.00 . The formula of $\chi^{2}$ minimization is

$$
\chi^{2}\left(H_{0}, \Omega_{M}, \alpha, \eta\right)=\sum_{i}\left[\frac{\mu\left(H_{0}, \Omega_{M}, \alpha, \eta ; z_{i}\right)-\mu_{\mathrm{obs}}\left(z_{i}\right)}{\sigma\left(z_{i}\right)}\right]^{2}
$$

where $u\left(H_{0}, \Omega_{M}, \alpha, \eta ; z\right)$ is obtained from Equation (4.1d), and $u_{\mathrm{obs}}$ is the observational value of SNe Ia with error $\sigma\left(z_{i}\right)$ in Union2.1.

In the analysis, we marginalize $\Omega_{M}$ by integrating over them, and select $H_{0}=70 \mathrm{kms}^{-1} \mathrm{Mpc}^{-1}$. There are some things need to be emphasized. Firstly, there is no Gaussian prior on $\Omega_{M}$, and we just assume that $\Omega_{M}$ has a uniform distribution in interval $[0,1]$. Secondly, we must select $H_{0}=70 \mathrm{kms}^{-1} \mathrm{Mpc}^{-1}$ in our analysis, because the distance modulus of Union2.1 compilation is dependent on $H_{0}$, and the Union2.1 sample assumed $H_{0}=70 \mathrm{kms}^{-1} \mathrm{Mpc}^{-1}$ to get the distance modulus of SNe Ia (see Section 3).

We assume that $\eta$ is a constant, i.e.,

$$
\eta=1+\delta
$$

where $\delta$ is a little constant. When $\delta=0$, the DD relation is satisfied.

By marginalizing $\Omega_{M}$, we obtain that the best values of $\alpha$ and $\delta$ are 0.93 and 0 respectively. The two-dimensional confidence regions of $\alpha$ and $\delta$ are shown in Figure 1(a). 


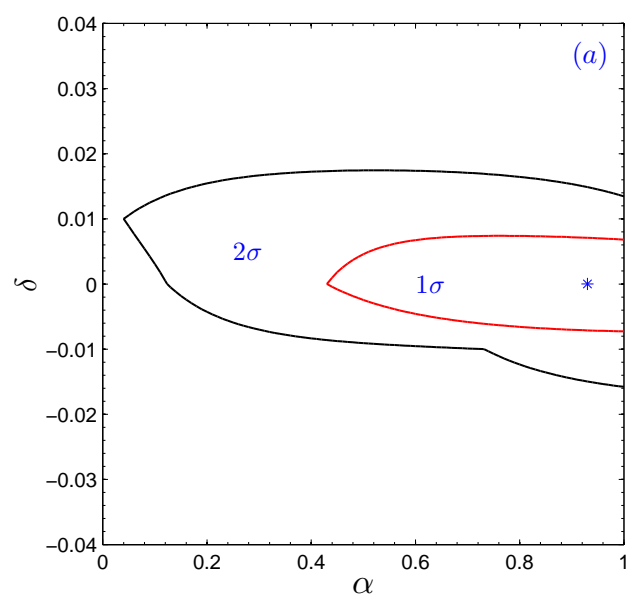

Figure 1. (a): Confidence regions at $68.3 \%$ and $95.4 \%$ levels from inner to outer respectively on the $(\alpha, \delta)$ plane for the sample Union2.1. The "*" in the center of confidence regions indicates the best fitted values $(0.93,0)$.
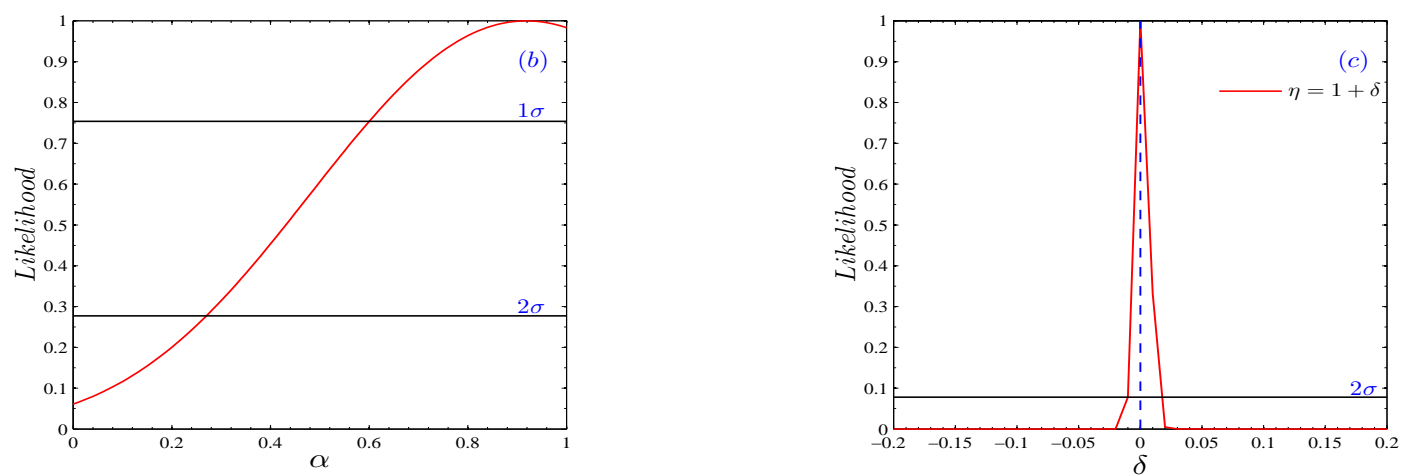

Figure 2. (b): PDF for $\alpha$. (c): PDF for $\delta$. Obviously, the D-R equation is in good consistency with the DD relation $(\delta=0)$. The step length of $\delta$ is 0.01 in our analysis, which is larger than the range of values for $\delta$ at $1 \sigma$, so we do not plot $1 \sigma$ confidence interval of $\delta$ on the picture.

In Figure 2 (b) and (c), the one-dimensional probability distribution of $\alpha$ and $\delta$ are plotted respectively. Figure 2(b) shows that $\alpha=0.92_{-0.32}^{+0.08}$ at $1 \sigma$ and $0.92_{-0.65}^{+0.08}$ at $2 \sigma$. For Figure 2(c), the result is $\delta=0_{-0.01}^{+0.01}$ at $2 \sigma$. The step length of $\delta$ is 0.01 in our analysis, which is larger than the range of values for $\delta$ at $1 \sigma$, so we do not give $1 \sigma$ confidence interval of $\delta$. Because the $\mathrm{DD}$ relation is valid when $\delta=0$, these results display that the $\mathrm{D}-\mathrm{R}$ approximation equation is in consistency with the DD relation. For a inhomogeneous universe which is described by Riemannian geometry, the DD relation should be met. Therefore, the D-R approximation equation is well if we use the DD relation to weigh it. 


\section{Discussions and Conclusions}

With the improvement of precision of observations in astronomy, accurate estimate of the distance is requested. Therefore, many theories which describe the inhomogeneity have been proposed to correct the distance-redshift relation [37]. Such as, LTB model [38], swisscheese model [4], weak-lensing approximation [39], and Dyer-Roeder relation [40]. Among them, the Dyer-Roeder equation is the simplest distance estimator, because it is obtained by adding small perturbation on FLRW model. Therefore, constraining $\alpha$ from D-R equation is essential.

In this article, we use the latest Union2.1 SNe Ia sample to constrain $\alpha$. We find that $\alpha \geq 0.60$ at $1 \sigma, \alpha \geq 0.27$ at $2 \sigma$, and the best fitted value of $\alpha$ is 0.92 . The D-R equation will reduce to general distance-redshift relation of $\Lambda \mathrm{CDM}$ model if $\alpha=1$, so our results show that the distance-redshift equation of $\Lambda \mathrm{CDM}$ model is not the best equation for distance. Furthermore, the D-R approximate equation is a perturbation equation. Whether it put a disturbance on the DD relation need to test. Our results illustrate that the D-R equation does not violate the $\mathrm{DD}$ relation. So the $\mathrm{D}-\mathrm{R}$ approximation equation is well if we use the DD relation to weigh it.

Of course, $\alpha$ should not be a constant, but a function of $z$ [7]. However, Current data is not enough to study its functional forms $\alpha(z)$. Bolejko. K [26] studied the relation between $\mathrm{D}-\mathrm{R}$ approximation equation and weak-lensing approximation, and they concluded that the two approximations could be compatible when $[\alpha(z)-1] \sim 1 /(1+z)^{5 / 4}$. In the future, with the increase of high-redshift SNe Ia data, the approximate functional forms of $\alpha(z)$ may be determined, and then, the estimate of distance will be more accuracy.

\section{Acknowledgments}

X. Y. is very grateful to Jian-Chuan Zheng, Ji-Long Chen, Zhong-Xu Zhai, Cong Ma and Shuo Yuan for their kind help. This work was supported by the National Science Foundation of China (Grants No. 11173006), the Ministry of Science and Technology National Basic Science program (project 973) under grant No. 2012CB821804, and the Fundamental Research Funds for the Central Universities.

\section{References}

[1] S. Weinberg, Cosmology, Oxford University Press, Oxford (2008)

[2] B. Bertotti, The Luminosity of Distant Galaxies, Proc. R. Soc. London A 294 (1966) 195

[3] J. E. Gunn, On the Propagation of Light in Inhomogeneous Cosmologies. I. Mean Effects, Astrophys. J. 150 (1967) 737

[4] R. Kantowski, Corrections in the Luminosity-Redshift Relations of the Homogeneous Fried-Mann Models, Astrophys. J. 155 (1969) 89

[5] Ya. B. Zeldovich, Observations in a Universe Homogeneous in the Mean, Soviet Astronomy 8 (1964) 13

[6] C. C. Dyer and R. C. Roeder, Distance-Redshift Relations for Universes with Some Intergalactic Medium, Astrophys. J. 180 (1973) L31

[7] R. C. Santos, J. V. Cunha and J. A. S. Lima, Constraining the dark energy and smoothness-parameter with supernovae, Phys. Rev. D 77 (2008) 023519 [arXiv:0709.3679v2] 
[8] A. G. Riess, L. G. Strolger, S. Casertano et al., New Hubble Space Telescope Discoveries of Type Ia Supernovae atz > 1: Narrowing Constraints on the Early Behavior of Dark Energy, Astrophys. J. 659 (2007) 98 [arXiv:astro-ph/0611572v2]

[9] H. R. Yu, T. Lan, H. L. Wan, T. J. Zhang and B. Q. Wang, Constraints on smoothness parameter and dark energy using observational $H(z)$ data, Research in Astron. Astrophys. 11 (2011) 125 [arXiv:1008.1935v1]

[10] V. c. Busti, R. C. Santos and J. A. S. Lima, Constraining the dark energy and smooth parameter with type Ia supernovae and Gamma-Ray Bursts, Phys. Rev. D 85 (2012) 103503 [arXiv:1202.0449]

[11] G. F. R. Ellis, On the definition of distance in general relativity: I. M. H. Etherington, Gen. Rel. Grav. 39 (2007) 1047

[12] I. M. H. Etherington, On the Definition of Distance in General Relativity, Phi. Mag. 151933 761

[13] G. F. R. Ellis, Republication of: Relativistic cosmology, Gen. Rel. Grav. 41 (2009) 581

[14] R. F. L. Holanda, J. A. Lima and M. B. Ribeiro, Testing the Distance-Duality Relation with Galaxy Clusters and Type Ia Supernovae, Astrophys. J. 722 (2010) L233 [arXiv:1005.4458v2]

[15] R. F. L. Holanda, J. A. Lima and M. B. Ribeiro, Cosmic Distance Duality Relation and the Shape of Galaxy Clusters, Astron. Astrophys. 528 (2011) L14 [arXiv:1003.5906v2]

[16] J. P. Uzan, N. Aghanim and Y. Mellier, The distance duality relation from X-ray and $S Z$ observations of clusters, Phys. Rev. D 70 (2004) 083533 [arXiv:astro-ph/0405620v1]

[17] B. A. Basset and M. Kunz, Cosmic distance-duality as probe of exotic physics and acceleration, Phys.Rev. D 69 (2004) 101305 [arXiv:astro-ph/0312443v2]

[18] Z. Li, P. Wu and H. Yu, Cosmological-model-independent tests for the distance-duality relation from Galaxy Clusters and Type Ia Supernova, Astrophys. J. 729 (2011) L14 [arXiv:1101.5255v2]

[19] X. L. Meng, T. J. Zhang, H. Zhan and X. Wang, Morphology of Galaxy Clusters: A Cosmological Model-Independent Test of the Cosmic Distance-Duality Relation, Astrophys. J. $\mathbf{7 4 5}$ (2012) 98 [arXiv:1104.2833v2]

[20] E. De Fillips, M. Sereno, M. W. Bautz and G. Longo, Measuring the Three-Dimensional Structure of Galaxy Clusters. I. Application to a Sample of 25 Clusters, Astrophys. J. 625 (2005) 108 [arXiv:astro-ph/0502153v1]

[21] J. A. S. Lima, J. V. Cunha and V. T. Zanchin, Deformed Distance Duality Relations and Supernovae Dimming, Astrophys. J. 742 (2011) L26 [arXiv:1110.5065v1]

[22] A. Avgoustidis, C. Burrage, J. Redondo, L. Verde. and R. Jimenez, Constraints on cosmic opacity and beyond the standard model physics from cosmological distance measurements, JCAP 10 (2010) 024 [arXiv:1004.2053v1]

[23] F. De Berbardis, E. Giusarma and A. Melchiorri, Constraints on Distance Duality Relation from Sunyaev Zel'dovich Effect and Chandra X-ray measurements, Int. J. Mod. Phys. D 15 (2006) 759 [arXiv:gr-qc/0606029v1]

[24] R. Sachs, Gravitational Waves in General Relativity. VI. The Outgoing Radiation Condition, Royal Society of London Proceedings Series A 264 (1961) 309

[25] E. V. Linder, Light propagation in generalized Friedmann universes, Astron. Astrophys. 206 (1988) 190

[26] K. Bolejko, Weak lensing and the Dyer-Roeder approximation, MNRAS 412 (2011) 1937 [arXiv:1011.3876v1] 
[27] E. V. Linder, Transition from Clumpy to Smooth Angular Diameter Distances, Astrophys. J. 497 (1998) 28 [arXiv:astro-ph/9707349v2]

[28] S. Räsänen, Light propagation in statistically homogeneous and isotropic dust universes, JCAP 02 (2009) 011 [arXiv:0812.2872v2]

[29] R. C. Santos and J. A. S. Lima, Clustering, Angular Size and Dark Energy, Phys. Rev. D 77 (2008) 083505 [arXiv:0803.1865v1]

[30] R. Kantowski, The Effects of Inhomogeneities on Evaluating the mass parameter $\Omega_{m}$ and the cosmological constant $\Lambda$, Astrophys. J. 507 (1998) 483 [arXiv:astro-ph/9802208v2]

[31] R. Kantowski, J. K. Kao and R. C. Thomas, Distance-Redshift Relations in Inhomogeneous Friedmann-Lemaitre-Robertson-Walker Cosmology, Astrophys. J. 545 (2000) 549

[32] R. Kantowski and R. C. Thomas, Distance-Redshift in Inhomogeneous $\Omega_{0}=1$ Friedmann-Lemaitre-Robertson-Walker Cosmology, Astrophys. J. 561 (2001) 491 [arXiv:astro-ph/0011176v2]

[33] M. Demianski, R. de Ritis, A. A. Marino and E. Piedipalumbo, Approximate angular diameter distance in a locally inhomogeneous universe with nonzero cosmological constant, Astron. Astrophys. 411 (2003) 33 [arXiv:astro-ph/0310830v1]

[34] N. Suzuki, D. Rubin, C. Lidman et al., The Hubble Space Telescope Cluster Supernova Survey: $V$. Improving the Dark Energy Constraints Above $z>1$ and Building an Early-Type-Hosted Supernova Sample, Astrophys. J. 746 (2012) 85 [arXiv:1105.3470v1]

[35] R. Amanullah, C. Lidman, D. Rubin et al.,Spectra and Hubble Space Telescope Light Curves of Six Type Ia Supernovae at $0.511<z<1.12$ and the Union2 Compilation, Astrophys. J. 716 (2010) 712 [arXiv:1004.1711v1]

[36] J. Guy, P. Astier, D. Hardin et al., SALT2: using distant supernovae to improve the use of Type Ia supernovae as distance indicators, Astron. Astrophys. 466 (2007) 11 [arXiv:astro-ph/0701828v1]

[37] K. Bolejko, M. N. Célérier and A. Krasiński, Inhomogeneous cosmological models: exact solutions and their applications, Class. Quantum Grav. 28 (2011) 164002 [arXiv:1102.1449]

[38] M. N. Célérier, Do we really see a cosmological constant in the supernovae data? Astron. Astrophys. 353 (2000) 63 [arXiv:astro-ph/9907206]

[39] T. Pyne and M. Birkinshaw, The luminosity distance in perturbed FLRW space-times, MNRAS 348 (2004) 581 [arXiv:astro-ph/0310841]

[40] C. C. Dyer and R. C. Roeder, The Distance-Redshift Relation for Universes with no Intergalactic Medium, Astrophys. J. 174 (1972) L115 